\title{
COMMISSION 28: GALAXIES
}

\section{Report of Meetings, 19 and 20 and 21 August 1970}

\author{
President: G. C. McVittie \\ SECRETARY: E. B. Holmberg
}

The first session dealt with business matters, the second and third sessions with presentation and discussion of a series of papers on extragalactic research. In addition, a joint meeting with Commission 40 was held on 20 August.

\section{Business Meeting}

The printed Report presented by the President on research work in the extragalactic field during the past three years was gratefully acknowledged by the Commission. A number of members expressed the wish that in future reports the abbreviations of titles of scientific periodicals should conform to the rules previously established by the IAU.

The President announced that in its first session on 18 August the General Assembly had decided to create two new Commissions: No. 47 on Cosmology, and No. 48 on High Energy Astrophysics. The Commission expressed its approval of this decision, on the condition that, if possible, separate meetings of Commissions 28 and 47 never be arranged simultaneously.

The President proposed, and the Commission approved, the list of names to be sent to the Executive Committee of the Union and those of persons who should form the Organizing Committee of the Commission.

On account of the difficulties in defining the boundary-lines between Commissions 28 and 47, the members of Commission 28 expressed the wish that the new President ask each individual member about his (her) preference as regards membership of the two Commissions.

The Commission accepted the proposal of Dr Zwicky that the Working Group on Supernovae should transfer from Commission 28 to Commission 27.

The business meeting was concluded by a discussion of general rules of proceedings that might possibly be established for future meetings of the Commission.

\section{First Scientific Meeting}

At this session the following seven papers were presented:

1. F. Zwicky: Compact Galaxies.

2. V. A. Ambarcumjan: Galaxies with Ultra-Violet Excess.

3. G. O. Abell: Giant Galaxies in Clusters.

4. J. Heidmann: Diameter-Luminosity Relation and Distance of the Virgo Cluster.

5. A. K. Alksnis: Novae in Outer Regions of M 31.

6. J. D. Wray: Velocity Field of Ionized Gas in M 82.

7. H. Oleak: Radio Emission from Supernova Remnants in Distant Galaxies.

Each presentation was followed by a short period of discussion.

\section{Second Scientific Meeting}

At the final session the following eight papers were presented:

8. W. A. Baum: Diameter-Redshift Relation.

9. K.-H. Schmidt: On the Mass Discrepancy of Clusters of Galaxies.

10. L. Bottinelli: The Large-Scale Spatial Distribution of Neutral Hydrogen in Galaxies. 
11. J. E. Ejnasto: Proposal for 'Complex Investigation of the Galaxy and Nearby External Galaxies'.

12. M. H. J. Demoulin: Remarks on Markarian Galaxies.

13. H. C. Arp and F. Bertola: The Large Optical Extensions of Elliptical Galaxies.

14. G. de Vaucouleurs: Inclination Effects in Apparent Diameters of Galaxies.

15. A. Przybylski: Analysis of HD 32034 in LMC.

As before, each paper was followed by a short period of discussion.

\section{Reports of Working Groups}

The three Working Groups of Commission 28 have submitted the following reports on their activities.

The Working Group on Galaxy Photometry, with G. de Vaucouleurs as chairman, held two separate meetings in which reports and papers were presented by J. D. Wray, E. Vandekerkhove, P. Wild, G. de Vaucouleurs, J. E. Solheim, G. O. Abell, C. Fraser, F. Bertola, A. G. Pacholczyk, and P. D. Usher. The reconstituted membership consists of the following: G. O. Abell, H. D. Ables, G. F. Benedict, F. Bertola, J. H. Bigay, T. M. Borchkadze, W. Bronkalla, G. de Vaucouleurs, S. D'Odorico, C. Fraser, P. W. Hodge, E. B. Holmberg, E. K. Kharadze, E. J. Kibblewhite, I. R. King, G. E. Kron, C. D. Mackay, B. E. Markarjan, R. H. Miller, P. Nilson, H. Oleak, G. Paal, N. Richter, H. J. Rood, J. L. Serrsic (chairman), C. D. Shane, R. R. Shobbrook, J. E. Solheim, D. W. N. Stibbs, P. D. Usher, E. Vandekerkhove, S. Van den Bergh, B. A. Voroncov-Vel'jaminov, P. Wild, J. D. Wray.

The Working Group on Supernovae, with F. Zwicky as chairman, held one meeting, at which the decision was taken to accept the invitation of Commission 27 to transfer the Working Group from Commission 28 to Commission 27. Short reports were presented by L. Rosino, A. D. Thackeray, L. Detre, J. A. Hynek, and P. Wild; recent results and future plans were briefly summed up by F. Zwicky. The reconstituted membership consists of the following: V. A. Ambarcumjan, Ch. Bertaud, F. Bertola, A. A. Boyarchuk, E. Chavira, L. Detre, G. Haro, J. A. Hynek, E. K. Kharadze, B. V. Kukarkin, H. Lambrecht, J. B. Oke, L. Rosino, W. L. Sargent, J. L. Serrsic, A. D. Thackeray, B. E. Westerlund, P. Wild, F. Zwicky (chairman).

The Working Group on the Magellanic Clouds met on 22 August 1970 and short contributions were read from the following authors: J. Graham, P. A. Wayman, W. Tifft, T. Schmidt, Ch. Fehrenbach, A. Ardeberg, M. Walker, M. W. Feast, T. Lloyd Evans and J. Dachs.

The reconstituted membership of the Group is as follows: A. D. Thackeray (chairman), V. M. Blanco, J. Landi Dessy, Ch. Fehrenbach, S. C. B. Gascoigne, P. W. Hodge, G. de Vaucouleurs, P. A. Wayman, B. E. Westerlund.

With regard to future work, the need for regular nova searches in the Clouds, especially with objective prism, was stressed. It is probable that many novae occur without detection. 